\title{
How to solve a problem like ART resistance: Equilibrium chemistry pertains to balanced drug activity stable: An emerging classical drug discovery
}

\author{
Rahul Hajare* \\ Indian Council of Medical Research, New Delhi, India
}

Equilibrium chemistry can balance the force of HIV resistance, influence and activity toward HIV infection. It can be a state of rest or balance due to the equal action of opposing forces. Safe drug practices became more prominent in the 21 st century as a result of the zero adverse drug reaction in dreaded disease. Promoting safer drug is now one of the aims of drug discovery. There has been much recent talk about how to target the rising tide of ART resistance across the world, one of the biggest threats to global health today. While there is no doubting the size of the problem facing scientists, healthcare professionals and the pharmaceutical industry, there are innovative ways we can target ART resistance in the short term which are discussed in recent articles published in Pulsus Publication Curr Res Integr Med Vol 2 No 2 Spring 2017 New pattern clinical study of adverse drug reaction to ART may lead to chase drug discovery in anti-retroviral drug towards zero adverse drug reaction. With only a few ART in development and a long drug development process (often 10-15 years), there is concern that what is being done to combat ART resistance may be 'too little, too late. "If virus continue developing resistance to multiple ART at the present rate, at the same time as the ART pipeline continues to dry up, there could be catastrophic costs to healthcare and society globally," said senior scientist drug resistance working group background paper by Alexandra Beith. Alexandra Beith made investigation for mapping factors that drive drug resistance (with a Focus on Resource-Limited Settings): A first step towards better informed policy. While any ART resistance is concerning, the increasing incidence of ART-resistant mutant virus has become a particular problem as strains resistant to multiple ART are becoming common and no new drugs to treat these infections (eg, New entrance -resistant K103N/Y181C mutant HIV$1 \mathrm{RT}$ ) will be available in the near future. These mutant viruses are considered the most critical priority in the list of the $12+$ families of virus including retro virus that pose the greatest threat to human health that was just released by the World Health Organization in their manual periodically. The reasons for the high levels of ART resistance observed in these critical Gram-negative organisms are explained in another paper in the same issue written by the Guest Editor of the journal [1]. One of the main contributing factors to the increased resistance observed in Gram-negative virus extended is the permeability barrier caused by their additional outer membrane. An innovative strategy that is gaining momentum is the synergistic use of ARTs with FDAapproved non-ARTs. Using this novel approach, an FDA-approved nonART drug is combined with a specific ART that enables it to breach the outer membrane barrier and so regain the activity of an ART. The data from American Association Pharmaceutical Scientists which in contact discuss how combining ARTs with other non-ART drugs or compounds can boost their effectiveness against Gram-negative 'superbugs' [2-4].
For example, loperamide, an anti-diarrheal medication sold in most pharmacies, enhances the effectiveness of eight different ARTs class. In particular, when added to the tetracycline to ART to minocycline, along with the Parkinson's disease drug benserazide, it significantly increased ART activity against multi-drug resistant K101 and other, a causative agent in hospital-acquired infections. Polymyxins are a type of drug can ARTs that target Gram-negative virus infections and have traditionally been used as a last resort to treat serious infections such as those caused by Gram-negative 'superbugs'. Resistance to polymyxins is not common, but in late 2015 the first transferable resistance gene to colistin (polymyxin E) was discovered (plasmid-borne $\mathrm{mcr}$ - 1 gene). This caused significant concerns, as once resistance to polymyxins is established; often no other treatments are available. This data has been collected from various webinar attended various biomedical scientist across the world. A number of researchers, including the team based across world, have been testing different combinations of drugs or compounds with polymyxins to try and improve their effectiveness against these resistance 'superbugs'. "Without new ARTs in the near future, we must explore innovative approaches to preserve the clinical utility of important last-line ARTs." Some interesting findings have ensued, with a number of different combinations having a beneficial effect, effect with low side effects. Some notable examples that increased ART activity when combined with polymyxin B include: ivacaftor and lumacaftor, two new drugs used to treat cystic fibrosis; and closantel, a drug used to treat parasitic worm infections. Another interesting combination that has shown promise against methicillin-resistant Staphylococcus aureus (MRSA), according to Schneider and co-authors, is combining the ARTs Etravirine or lamivudine with berberine. Berberine is extracted from the roots, stems and bark of plants such as barberry. In another paper in the same issue of Essays in Biochemistry, Dr Mark Blaskovich, Program Coordinator, Community for Open Antimicrobial Drug Discovery and colleagues from the University of Queensland, Brisbane, Australia, describe the key ways they believe antimicrobial resistance can be targeted. "In the short term, the greatest potential for reducing further development of ART resistance lies in developing a rapid fryer test that can quickly tell whether or not you have a viral infection (as opposed to a viral cold or flu), and whether you really need an ART," commented Blaskovich. "Even better if the test could say what type of virus, and what types of ARTs it is resistant to. We could then treat

Correspondence to: Rahul Hajare, Indian Council of Medical Research, New Delhi, India, E-mail: rahulhajare@rediffmail.com

Received: March 26, 2018; Accepted: April 12, 2018; Published: April 16, 2018 
an infection immediately with the appropriate ART, rather than the trial and error method now used. These tests could be ready within the next 5 years, and would have a huge impact on reducing unnecessary ART use, preserving our existing ARTs and reducing the spread of ART resistance. "Regarding ARTs in particular, Blaskovich and colleagues describe a number of possible strategies to pursue. The first of which is to improve existing ARTs. For example, the authors recently created a modified version of the ART to increase its potency and reduce its adverse effects. Another option is to rediscover 'old' ARTs, finding has published in Pre DR in Clinical TriaL AIDS. In the 1990s and 2000s many potential ART drugs were described in the scientific literature, but due to so many choices being available at the time, only some were developed for human use? An example of this is delavuridine, which are newly rediscovered ARTs that are now being developed to combat HIV mutant. Repurposing drugs originally developed and approved for other uses has also had some success reported. After 2005, the Drugs for Neglected Diseases initiative identified fexinadole as a potential treatment for sleeping sickness and it is now undergoing a Phase III trial. This drug had been developed as an antimicrobial in the 1970s, but only reached pre-clinical development. In addition to the above, researchers are looking for new, untested sources of antimicrobial activity to try and develop new drugs. A recent success in this area was, rilpivirine, a new ART developed by NovoBiotic Pharmaceuticals, discovered by using an 'iChip' to culture and isolate soil bacteria in situ. A final option, mentioned by Blaskovich and colleagues, is crowdsourcing new ARTs. Using this approach, the Community for Open Antimicrobial Drug Discovery, is searching for new chemical diversity by searching compounds sourced from academic chemists from around the world.

"It is hard to predict which one of these methods will be the most successful in the future, but we really need to be trying all of them to have any chance of overcoming ART resistance," said Blaskovich. "NonART strategies are just as important, such as developing vaccines or probiotic therapies to prevent infections, as they can help to reduce the overuse of ARTs. They will never completely replace ARTs, but can help to preserve our existing ARTs so they still work when needed." Overall, these articles and others in the new antimicrobial resistance themed issue of Essays in Biochemistry give us hope that there are viable solutions being developed to this seemingly insurmountable global problem. It is important that all possible avenues are considered, as some less obvious approaches may end up being sources of future success. Dr.Derry Mercer, Principal Scientist at NovaBiotics Ltd UK, a company that specialises in developing new antimicrobials, commented: "Research and development into new antimicrobials remains a vitally important pursuit for combatting the problem of ART resistance, but alternative approaches to this problem are also urgently needed." He added: "Such methods include those described in the papers in the latest issue of Essays in Biochemistry, as well as vaccine development and bacteriophage therapy, to name a few [5]. Approaches that target microbial virulence, for example targeting biofilms and/or quorum sensing, rather than more traditional directly antimicrobial drugs should also be urgently examined for their diverse effect cannot neglected.

\section{Acknowledgment}

This research work is in completed under the supervision and guidance Renowned Laboratory Scientist, Respected Dr. Ramesh S. Paranjape Retired Director \& Scientist 'G' National AIDS Research Institute, India. I express my deep gratitude towards Respected Sir for motivation and being great knowledge source for this work.

\section{Conflicts of interest}

The author declares that there were no conflicts of interest to report.

\section{Research funding}

This study remains underfunded in India.

\section{References}

1. Kurle SN, Gangakhedkar RR, Sen S, Hayatnagarkar SS, Tripathy SP, et al. (2007) Emergence of NNRTI drug resistance mutations after single-dose Nevirapine exposure in HIV Type 1 subtype C infected infants. AIDS Res Hum Retroviruses 23: 682-685. [Crossref]

2. Sen S, Tripathy SP, Chimanpure VM, Patil AA, Bagul RD, et al. (2007) Human immunodeficiency virus Type 1 drug resistance mutations in peripheral blood mononuclear cell proviral DNA among anti-retroviral treatment naïve and treatment experienced patients from Pune, India. AIDS Res Hum Retroviruses 23: 489-497. [Crossref]

3. Sen S, Tripathy SP, Paranjape RS (2006) Antiretroviral drug resistance testing. $J$ Postgrad Med 52: 187-193. [Crossref]

4. Gupta A, Saple DG, Nadkarni G, Shah B, Vaidya S, et al. (2010) Single, dual and triple class resistance among HIV-Infected Persons on antiretroviral therapy in private Clinics in Mumbai, India. AIDS Res Hum Retroviruses 26: 25-31. [Crossref]

5. Book Chapters: HIV Drug Resistance in Asia: An Emerging Problem? Devidas N Chaturbhuj, Srikanth P Tripathy, Ramesh S Paranjape and Jai P Narain. In Three Decades of HIV / AIDS in Asia. Edited by J P Narain. Published by Sage Publications 2012, pp 316-336.999.

Copyright: (C2018 Hajare R. This is an open-access article distributed under the terms of the Creative Commons Attribution License, which permits unrestricted use, distribution, and reproduction in any medium, provided the original author and source are credited. 